\section{EL DISEÑO DEL SISTEMA DE CERRAMIENTO}

CLAUDIO VÁSQUEZ Z .

Diversos conceptos se utilizan para caracterizar el sistema de cerramiento de los edificios. Al llamarlo piel, por ejemplo, se lo compara con la protección biológica de los cuerpos frente al medio externo. El término envolvente lo describe como aquello que protege algo interior, a la manera de una envoltura. Cuando se habla de fachada se refiere a su aspecto visual y compositivo; al hablar de cerramiento a su capacidad de contener y temperar.. Esta múltiple caracterización da cuenta de la complejidad inherente a su determinante rol espacial, es decir, la relación interior y exterior, que supone la vinculación de tres ámbitos que determinan sus condiciones de diseño: el exterior, el interior y el plano de cerramiento. La manera de relacionarse entre ellos determina en gran medida las condiciones de uso y confort interior, o desempeño del edificio.

Se abordará cada ámbito por separado para orientar respecto de las condiciones que cada uno impone al diseño de un sistema de cerramiento.

\section{EXTERIOR}

El ambiente exterior está determinado por el clima, que depende principalmente de las condiciones geofísicas y de la latitud del lugar donde se desarrolla un proyecto. Sus características se deducen del registro -durante FIG.01>

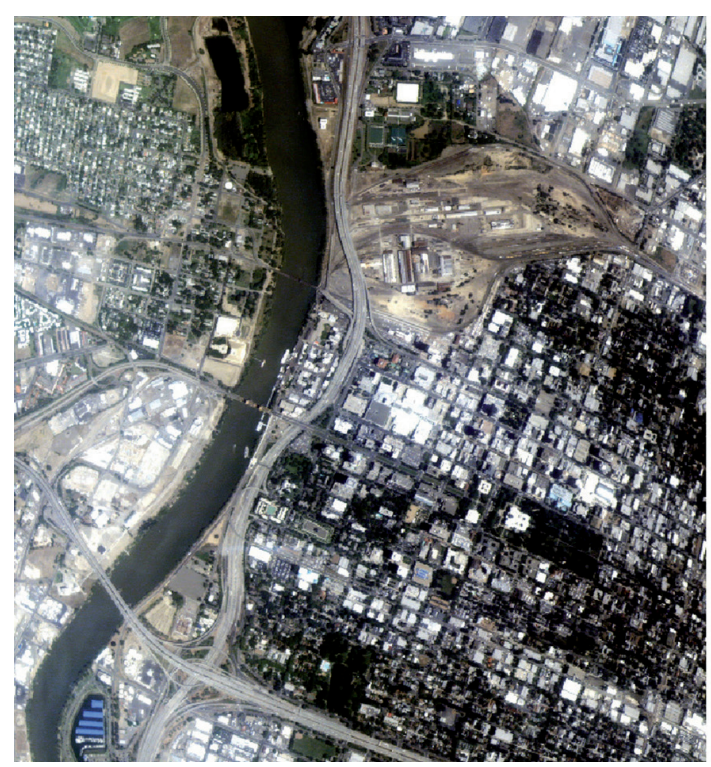

varias décadas- de sus condiciones meteorológicas, entre las cuales son relevantes para el diseño de un sistema de cerramiento: la radiación solar, la temperatura del aire, la humedad relativa y el viento ${ }^{1}$ (D’Alençon, 2008).

Si bien las características climáticas de un lugar son conocidas, no son suficientes por sí mismas. Para enfrentar el diseño de un sistema de cerramiento, porque hay factores propios del emplazamiento que los modifican. Es sabido que la acción humana ejerce influencias sobre las condiciones exteriores en las zonas urbanas, ya que estas se comportan como islas de calor -heat islands-por su diferencia tectónica con el medio natural circundante (FIG.01). Las superficies urbanas reciben radiación de onda corta y la reirradian en onda larga en función de su componente de cielo y de su albedo, pero también pueden reflejarla y aportar por esta vía energía a sus vecinos. El equilibrio entre ganancias y pérdidas determina el balance térmico urbano global según las particularidades de cada ciudad (Santamouris, 2001) y, por lo tanto, no es algo generalizable. Es así que al diseñar un edificio, no solamente es necesario considerar datos meteorológicos si no que es fundamental analizar el comportamiento del contexto inmediato, natural o artificial.

En general, las condiciones exteriores determinan la forma y materialidad global de los

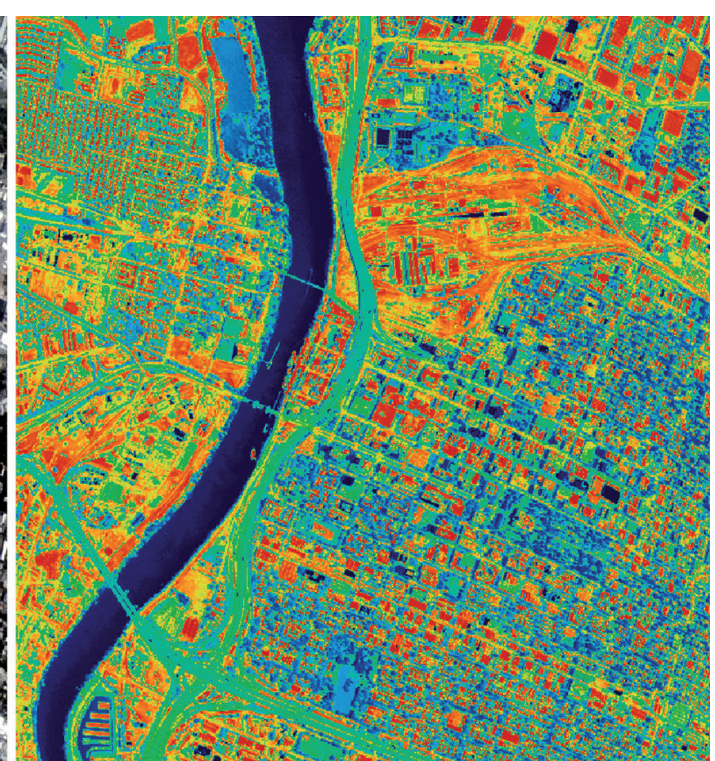

Claudio Vásquez Z. I Arquitecto y Magíster en Arquitectura, Pontificia Universidad Católica de Chile; Doctor Arquitecto, Universidad Politécnica de Cataluña, España. Profesor, investigador y coordinador de la linea de materialidad y sistemas, Escuela de Arquitectura, UC.

edificios, ya que hay mejores y peores maneras de responder a los diferentes climas. Por ejemplo, se considera adecuada una proporción en planta 1:2 a 1:3 en zonas cercanas al Ecuador, donde la posibilidad de interactuar con el exterior es deseable. Se tiende a proporciones más cuadradas en las latitudes más bajas, donde lo deseable es conservar y gestionar la energía interior. Por otra parte, se recomienda disponer el eje principal del edificio en sentido oriente-poniente, para que la fachada principal sea Norte o Sur, con el objetivo de minimizar la acción penetrante de la radiación solar proveniente del Oriente y del Poniente. Un edificio organizado longitudinalmente de Norte a Sur, puede llegar a consumir 1,5 veces lo que consume un edificio en igualdad de condiciones dispuesto de Oriente a Poniente (Yeang, 1999) (FIG.02).

En la optimización de las demandas de un edificio influye tanto la relación volumenenvolvente como su forma general. Un concepto asociado a la forma, y que permite prever su potencial consumo energético, es la compacidad que corresponde al cociente entre su volumen -potencial de energía almacenada-y la superficie de su envolvente - potencial intercambio energético interior-exterior-. Se ha demostrado que la potencial demanda de climatización es inversamente proporcional a la compacidad en climas fríos como el de París (Depecker, et ál., 2001). También se ha expuesto, a través de simulación en más de dieciocho mil casos, que un factor determinante para la selección de una u otra es el clima ${ }^{2}$ (Tiberiu, et ál., 2008). El inverso de la compacidad es el factor forma. En climas cálidos y húmedos se recomiendan factores forma elevados ( $\mathrm{f}>1,2$ ), que permitan grandes aberturas para facilitar la ventilación. En climas fríos, en cambio, se sugiere factores forma bajos que permitan aislar más fácilmente (Rey Martínez, 2006).

En síntesis, aquello que llamamos ámbito exterior determina las condiciones generales de la forma, sus proporciones y tamaño, cuestión que no es menor y que muchas veces resulta difícil por restricciones urbanísticas o normativas.

1 Para entender el clima en su totalidad, a los parámetros anteriores debe agregarse las precipitaciones y la nubosidad. 2 Se llama factor forma a la compacidad, cuestión que ocurre normalmente por tratarse de conceptos íntimamente relacionados; sin embargo, el cálculo que se hace corresponde a la compacidad. 
FIG.01 Sacramento, EE.UU. Comparación entre imagen fo tográfica y termográfica para demostrar su comportamiento térmico a modo de heat island. Fuente: Marshall Space Flight Center MSFC, NASA.

FIG.02 Un mismo volumen puede tener diferentes superficies de envolvente y con eso diferentes potenciales de intercambio con el exterior. Fuente: elaboración propia en base a Hegger.

FIG.03 Zona de confort de acuerdo a la temperatura del aire y la humedad relativa. Fuente: elaboración propia en base a Hegger.

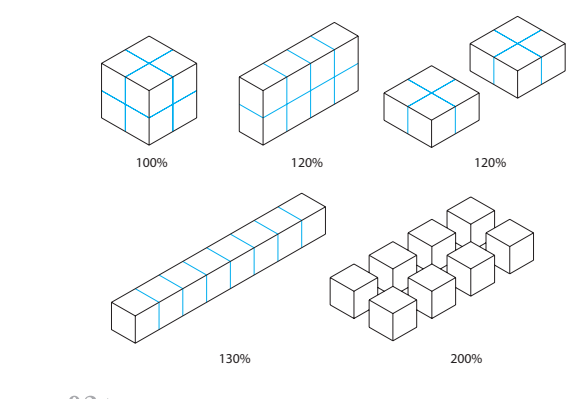

FIG.02 >

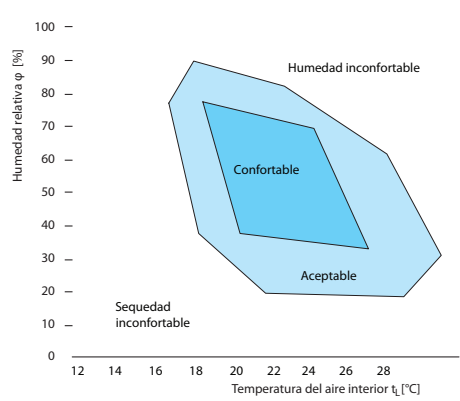

FIG.03>

INTERIOR

El objetivo del diseño del sistema de cerramiento es lograr interiores confortables de acuerdo al tipo de actividad que se realice en su interior, a través del control de los parámetros asociados al: confort térmico, visual y acústico. Especialmente es necesario evitar el disconfort térmico y el visual, porque ambos generan de forma automática consumos energéticos a través de los sistemas de climatización o iluminación artificial.

Confort térmico / Supone la coordinación de tres variables: temperatura, humedad y velocidad del aire. La primera depende de la temperatura del aire y del calor emitido por las superficies que rodean a las personas. La temperatura del aire es razonablemente uniforme, pero la de las superficies no, por lo tanto, en un mismo espacio pueden existir diferentes situaciones ambientales. Los gráficos muestran que el calor emitido por el suelo tiene menor amplitud que el cielo para generar situaciones confortables, consecuentemente, no da lo mismo en qué punto del espacio una persona se sitúe.

La humedad afecta la sensación de confort en función de la temperatura. Se considera
FIG.04 Zona de confort de acuerdo a la temperatura del aire y su velocidad. Fuente: elaboración propia en base a Hegger.

FIG.05 Balance térmico. Fuente: elaboración propia

LEYENDA

QT Flujo por transmisión

Qs Flujo por radiación sola

Qv Flujo por ventilación

QI Flujo por uso

QE Flujo por evaporación

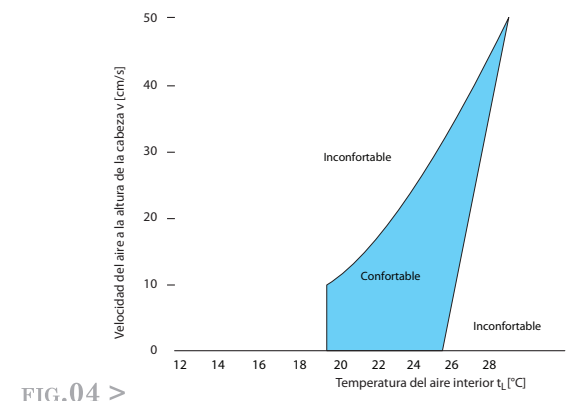

FIG.04 >

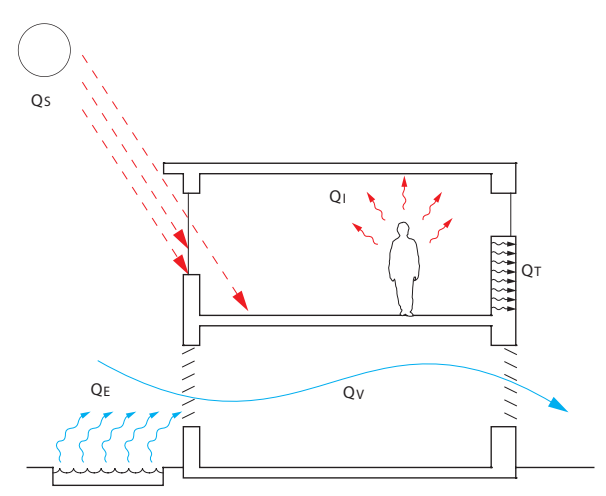

FIG. $05>$

que para temperaturas del aire entre $20 \mathrm{y}$ $22^{\circ} \mathrm{C}$, la sensación de confort se mantiene con humedades relativas entre 35 y 70 por ciento (FIG.03). La velocidad del aire afecta el balance energético de las personas porque disipa el calor corporal produciendo una sensación de confort, sin embargo, como muestra el gráfico, también existe una relación entre la velocidad del aire y la temperatura para que este fenómeno resulte aceptable (FIG.04).

Para mantener el interior en condición de confort es necesario conocer y manipular los flujos energéticos capaces de estabilizar las condiciones ambientales que lo determinan. La siguiente ecuación expresa las variables que están en juego para lograr dicha estabilidad (FIG.05): $\mathrm{QT}_{\mathrm{T}}+\mathrm{QS}+\mathrm{QV}+\mathrm{QI}+\mathrm{QE}=0$

La estabilidad del ambiente interior es un supuesto teórico, ya que todos los flujos anteriores están normalmente produciendo ganancias y pérdidas variables y, por lo tanto, el balance también varía. Cuando el resultado es positivo la temperatura asciende, y cuando es negativo, baja. La función de la climatización artificial es forzar los flujos para llevar la fluctuación
FIG.06 Esquema de funcionamiento de la ventilación nocturna. Fuente: elaboración propia.

FIG.07 Forma de abordar un eficiente flujo de ventilación natural interior. Fuente: elaboración propia.

FIG.08 Esquema de funcionamiento de una envolvente ventilada. Fuente: elaboración propia.

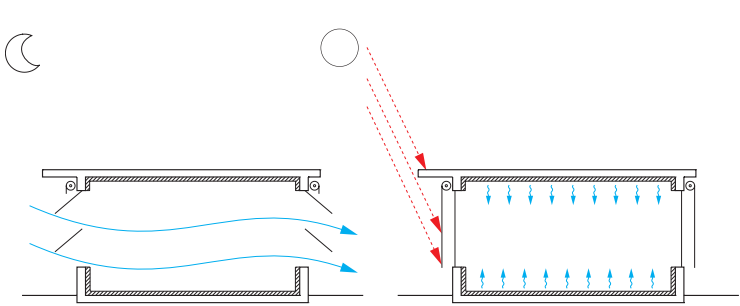

FIG.06 >
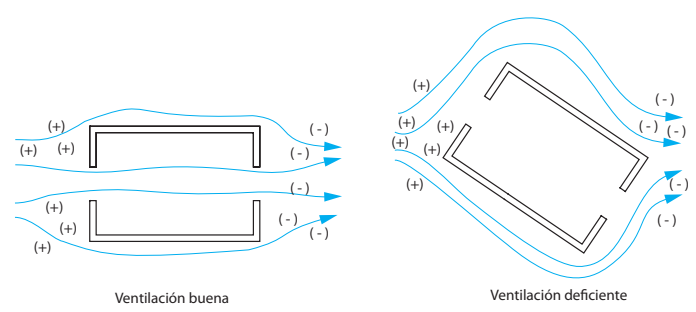

FIG.07 >

del balance a un rango donde la percepción del ambiente interior sea confortable. Para minimizar las demandas de energía y reducir las potencias de los equipos es necesario reprimir de forma pasiva uno o más de estos flujos.

En climas con variaciones amplias de temperatura diarias es posible utilizar la ventilación nocturna como estrategia de refrigeración pasiva. Esta consiste en el uso de materiales con una importante inercia térmica que se enfrían durante la noche a través de un flujo de aire (Qv) controlado. Así, a lo largo del día liberan la temperatura acumulada durante la noche. Para que la estrategia funcione los flujos de radiación solar (Qs) y ventilación $(\mathrm{Qv})$ natural deben ser minimizados de día, a través del aislamiento térmico exterior, usando protecciones solares y controlando las renovaciones de aire (FIG.06).

También es posible optimizar el flujo de ventilación $(\mathrm{Qv})$ mediante la disposición de las aperturas en concordancia con los vientos predominantes para forzar el paso y velocidad del aire por el interior, renovarlo y disipar su calor (FIG.07). 
FIG.09 Reacción de la luz frente a distintos tipos de superficie. Fuente: elaboración propia.

LEYENDA A y B: Reflexión

C: Refracción

FIG.10 Esquema de percepción de la luz. Fuente: elaboración propia.

FIG.11 Iluminancias de acuerdo al tipo de actividad. Fuente: DS 594 MINSAL

FIG.12 Espectro de diferentes tipos de sonidos. Fuente: elaboración propia.

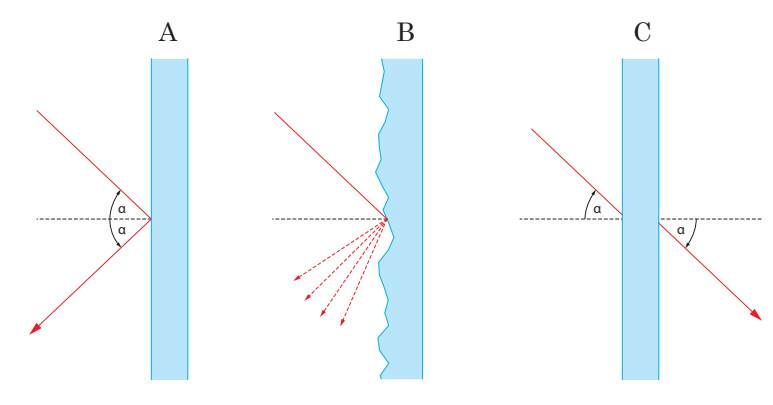

FIG.09 >

\begin{tabular}{ll}
\hline Lugar & lluminancia en Lux \\
\hline $\begin{array}{l}\text { Pasillos, bodegas, comedores. No } \\
\text { se necesita discriminación fina }\end{array}$ & 150 \\
\hline $\begin{array}{l}\text { Trabajo prolongado y leve. } \\
\text { Discriminación de detalles }\end{array}$ & 300 \\
\hline $\begin{array}{l}\text { Lectura continua, trabajos finos, } \\
\text { uso de herramientas }\end{array}$ & 500 \\
\hline $\begin{array}{l}\text { Laboratorios y salas de consulta } \\
\text { Costura, cortes y trazados }\end{array}$ & 500 a 700 \\
\hline $\begin{array}{l}\text { Trabajo prolongado y alta } \\
\text { discriminación de detalles }\end{array}$ & 1000 \\
\hline \begin{tabular}{l} 
Sillas dentales y de autopsias \\
\hline Mesa quirúrgica
\end{tabular} & 1500 a 2000 \\
\hline
\end{tabular}

FIG.11 >

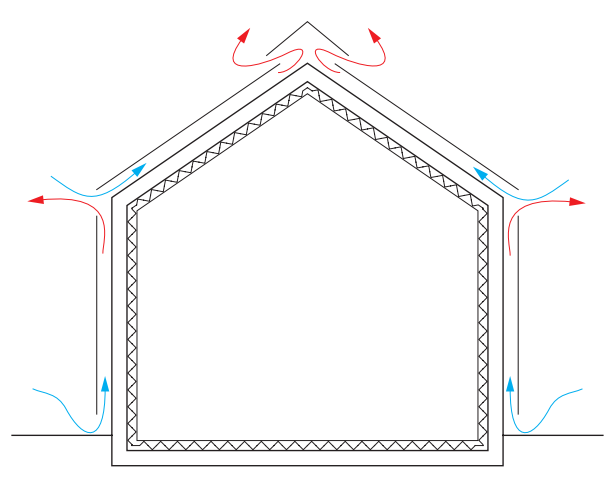

FIG.08 >

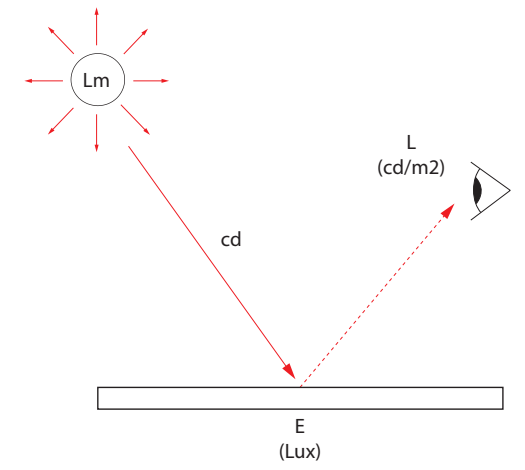

FIG. $10>$

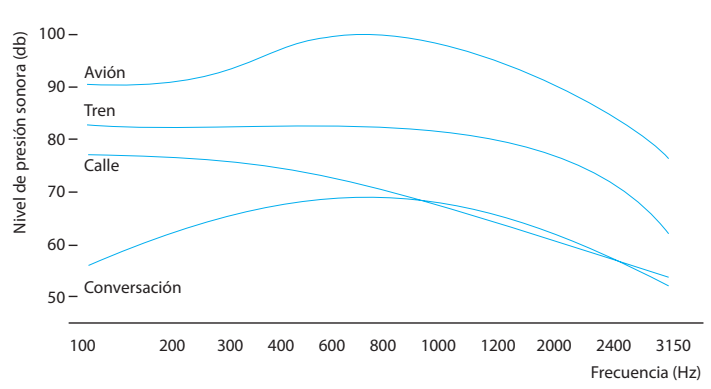

FIG.12 >
Para controlar el flujo de transmisión (QT) de las envolventes es posible usar una ventilada, que consiste en separar la envolvente térmica de la hidrófuga a través de una cámara de aire, que disipa el calor que genera el flujo de radiación solar (Qs) antes de que llegue a la envolvente en contacto directo con el espacio interior. El flujo por transmisión (QT) por lo tanto, disminuye (FIG.08).

Confort visual / La luz forma parte de la radiación solar y puede ser reflejada o refractada cuando incide sobre un cuerpo (FIG.09). Los cuerpos lisos y opacos la reflejan en un ángulo simétrico a la normal del plano sobre el cual incide (A); los opacos y rugosos la reflejan en múltiples direcciones (B); y los transparentes la refractan para transmitirla al interior $(\mathrm{C})$.

Considerando una fuente de luz dada -que puede ser el sol o un foco-, el flujo luminoso corresponde a la porción de energía radiante emitida por dicha fuente como luz, en todas direcciones y su unidad es el lumen (lm); por su parte la intensidad luminosa es el flujo en una dirección determinada y su unidad es la candela (cd). De ambas se deducen los dos conceptos básicos para entender el confort visual interior: la iluminancia, que es el flujo recibido por una superficie, cuyo símbolo es E y su unidad el lux $\left(\mathrm{lm} / \mathrm{m}^{2}\right)$; y la luminancia que es el flujo percibido por el ojo en una posición determinada con respecto al flujo en esa dirección, cuyo símbolo es L (cd/m²) (FIG.10).
Para lograr interiores lumínicamente confortables se busca que la iluminancia en el plano de trabajo sea suficiente para realizar la actividad en cuestión. Las iluminancias requeridas para cada actividad están normadas y pueden ser calculadas (FIG.11), sin embargo el problema es que la intensidad luminosa decrece en cada reflexión o refracción que la luz tiene en su tránsito desde la fuente al plano de trabajo. Por lo tanto su manejo depende de dos factores principales: tipo de superficie -determina cuánta luz y cómo será reflejada- y ángulo con que las distintas superficies conducen el flujo luminoso hasta llegar al plano de trabajo.

Entre los fenómenos que producen disconfort lumínico está el deslumbramiento, que no permite realizar las actividades normalmente puesto que el ojo solo se acostumbra a ambientes con luminancias uniformes. Este es un fenómeno común de los espacios interiores y se controla con filtros o pantallas que disminuyen la intensidad luminosa. Para lograr interiores bien iluminados, los suelos y los cielos deben ser considerados como planos lumínicamente activos, que pueden reforzarse con otras estrategias, como los conductos de sol -que permiten transportar luz hasta un punto deseado de la planta-, o las bandejas de sol, que se disponen en la fachada para reflejar el flujo luminoso hacia el cielo interior y lo sacan del campo visual.

Confort acústico / El sonido ${ }^{3}$ es un fenómeno mecánico producido por la vibración de un medio elástico, ya sea sólido, líquido o gaseoso, 


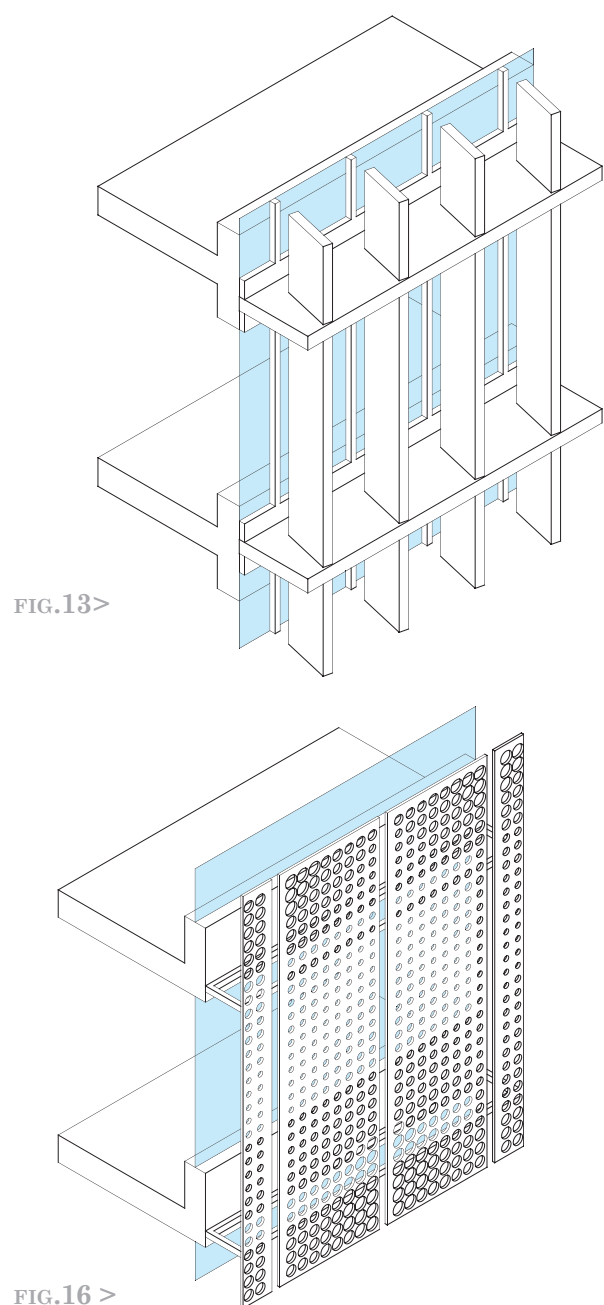

que produce variaciones de presión, medida en Pascales $(\mathrm{Pa})$, que se propagan en todas direcciones. El nivel sonoro expresa la intensidad vibratoria percibida por el oído y su unidad es el decibelio (dB). Un decibelio es la relación entre dos presiones: la recibida desde una fuente emisora y una presión de referencia que corresponde al umbral audible por el oído humano. Un sonido con una frecuencia de 0,00002 Pa equivale a $0 \mathrm{~dB}$, en cambio otro de $0,005 \mathrm{~Pa}$ equivale a $48 \mathrm{~dB}$.

Los sonidos están compuestos por un sinfín de intensidades y frecuencias caracterizables como un espectro. El gráfico (FIG.12) muestra el espectro de cuatro sonidos: un avión, un tren, una calle y una conversación; cada uno tiene una curva espectral propia.

Los medios de propagación del sonido en un edificio se pueden reducir a dos: el aire interior o exterior y los cuerpos sólidos o elementos constructivos que lo configuran.

Para la atenuación al sonido aéreo es determinante la masa de los elementos de la envolvente, ya que pierde energía de propagación de acuerdo a su frecuencia y la masa que lo atraviesa -Ley de Berger-. Por eso la selección de los materiales de la envolvente según su masa

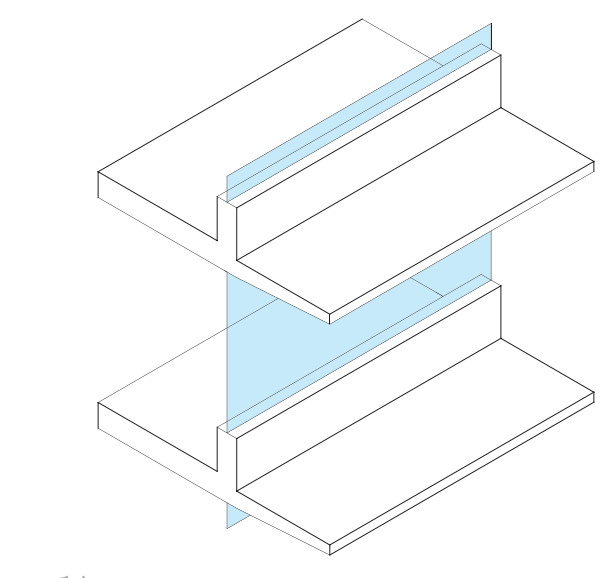

FIG.14 >

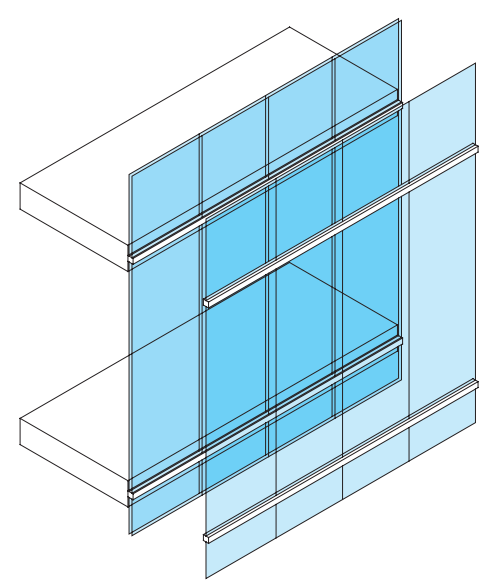

FIG.17 >

es una buena manera de aislar acústicamente un interior, utilizando discontinuidades para producir desacoplamientos.

El sonido de impacto se propaga a través de las estructuras de los edificios. No es posible romper su continuidad, por lo que para aislarlo se debe amortiguar la vibración en la propia fuente emisora. Esto porque cuando el sonido se irradia y llega llega a una superficie es difícil evitar su propagación de forma aérea.

\section{SISTEMA DE CERRAMIENTO}

Los sistemas de cerramiento deben tener la capacidad de controlar los diferentes flujos que pueden existir a través de ellos, para lo cual es necesario contar como mínimo con estrategias de control solar (Cs); control térmico $(\mathrm{CT})$; control lumínico (CL) y control acústico (CA).

Control solar (Cs) / Se refiere a la capacidad del cerramiento para controlar el flujo solar (Qs), que tiene directa consecuencia en el confort térmico y visual interior y depende de la radiación solar incidente en el lugar del proyecto. En general, es deseable minimizar el flujo solar (Qs) en verano, para disminuir las demandas de refrigeración, y maximizarlo en invierno para reducir la necesidad de calefacción.

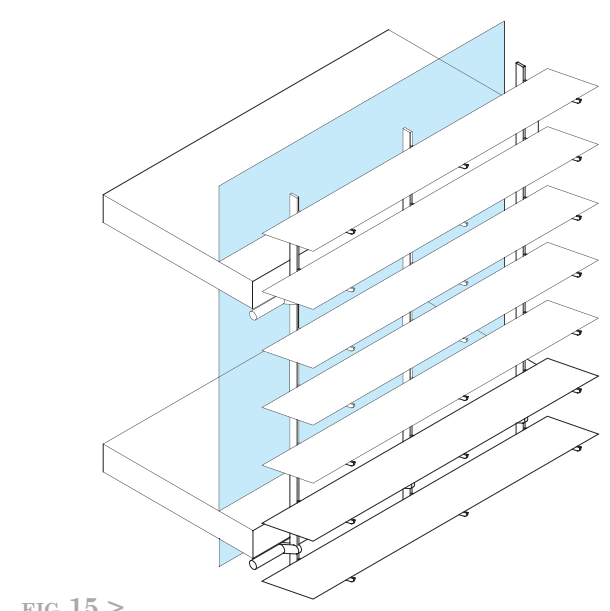

FIG.15 >

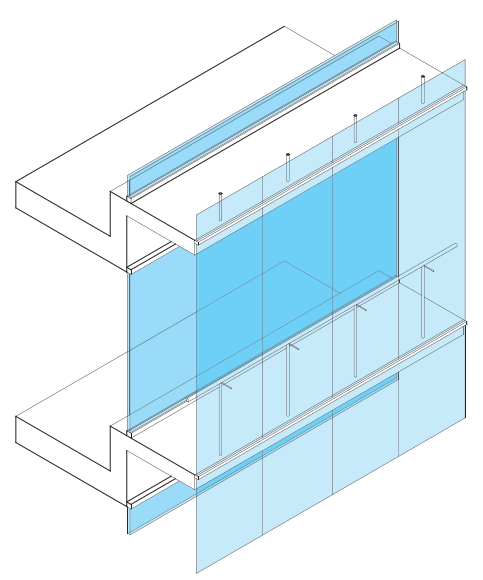

FIG.18 >

La estrategia más común utilizada para el control solar es el uso de protección solar, cuyo objetivo es ajustar el factor de sombra del sistema de cerramiento. De todas maneras sea cual sea el tipo usado, solo si se encuentra como primera capa exterior del cerramiento, será eficaz para el confort térmico. Cualquier sistema que se utilice en el interior, tendrá el rol principal de colaborar con el confort visual. Entre los elementos capaces de ejercer esta función se encuentran:

- Brise soleil: sistema de parasoles diseñados geométricamente, de acuerdo a la trayectoria solar, que se disponen en el exterior del sistema de cerramiento (FIG.13).

- Aleros: elementos que se proyectan horizontalmente y para controlar la radiación para ángulos solares máximos, cuya energía radiante es también máxima (FIG.14).

- Celosías: sistema de control solar basado en el tamizado de la radiación con el objetivo de mantener un componente de cielo activo en el interior (FIG.15).

- Pantallas: componentes dispuestos en un plano paralelo al sistema de fachada con el fin de bloquear la radiación solar directa. La densidad de la pantalla determina tanto la re- 

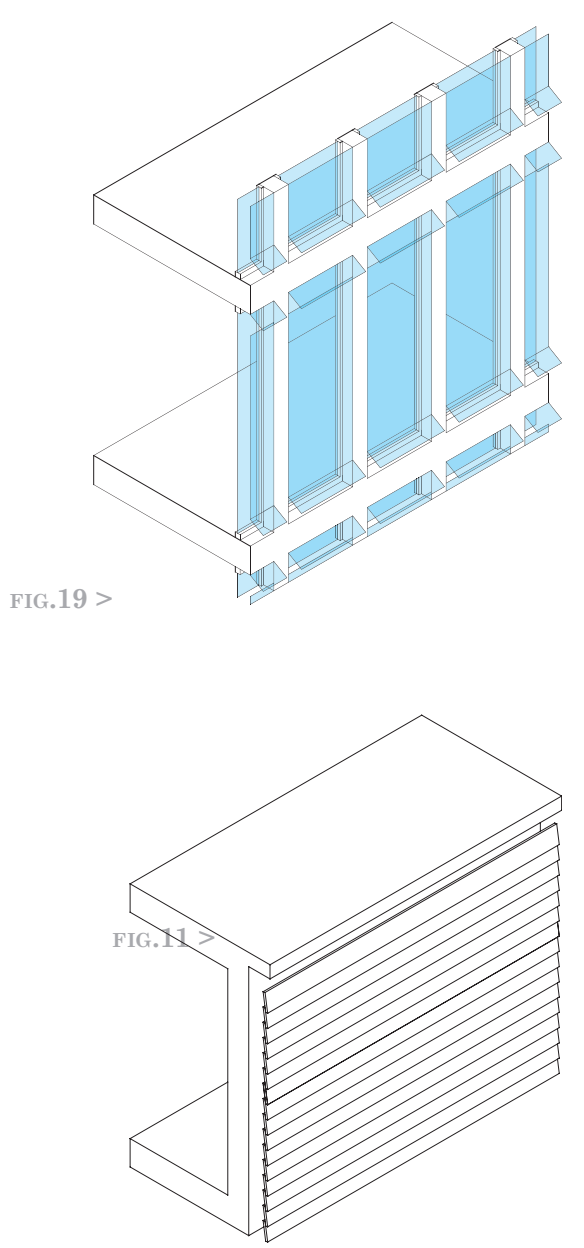

FIG.22 >
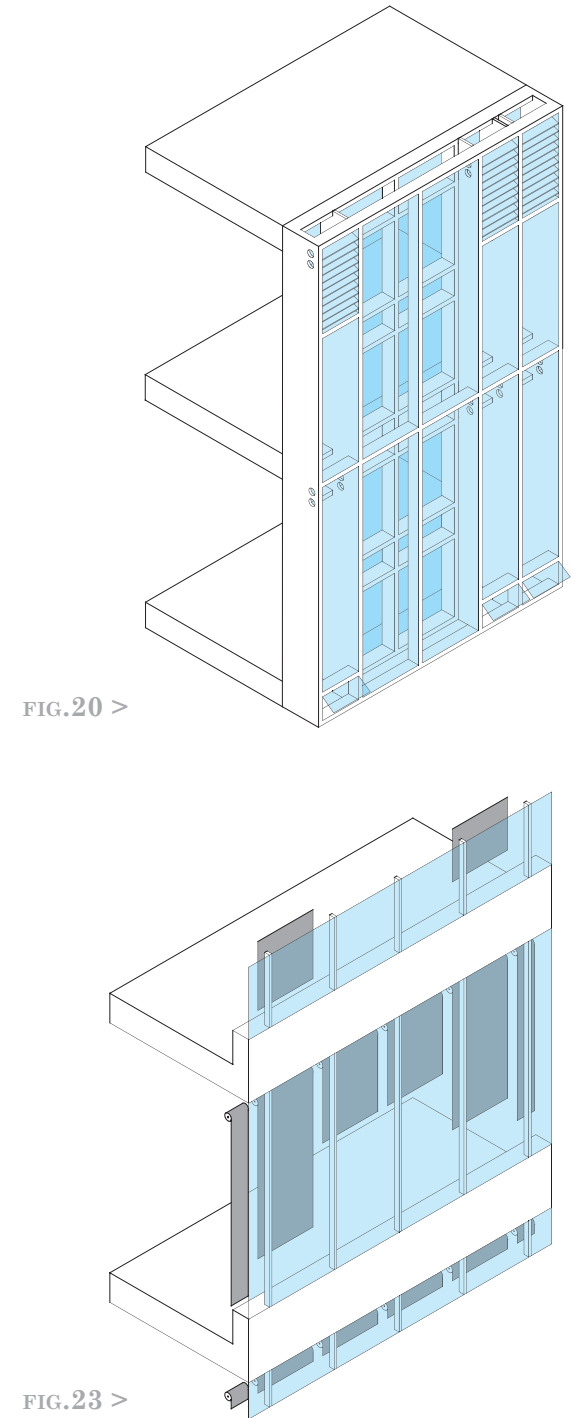

de aire, por lo que su uso eficiente se restringe a ciertos climas y a ciertas condiciones de gestión. Existen diferentes configuraciones que también responden a este principio (FIG.17).

- Doble piel corredor: se trata de una doble piel cerrada por cada piso para estimular que el aire fluya en sentido horizontal y así ventilar el espacio intermedio. Depende de la existencia de estructuras horizontales que también funcionan como parasoles (FIG.18).

- Doble piel cajón: en ella el aire ventila en un módulo de un vano, por lo tanto, la ventilación y la temperatura de la cámara se controla de forma localizada. Permite la ventilación del interior a través de la cámara (FIG.19).

- Doble piel shaft: doble piel cajón combinada alternadamente con módulos shafts de toda la altura de la fachada. Los cajones ventilan a través del shaft por convección (FIG.20).

- Muro trombe o fachada colectora: se utiliza el efecto invernadero para acumular calor en un muro de alta inercia térmica, el cual acumula calor y lo libera al interior en un régimen horario inverso al de la radiación (FIG.21). - Fachada ventilada: usa una cámara de aire sobrepuesta al cerramiento, cuyo flujo convectivo se estimula por la radiación solar que

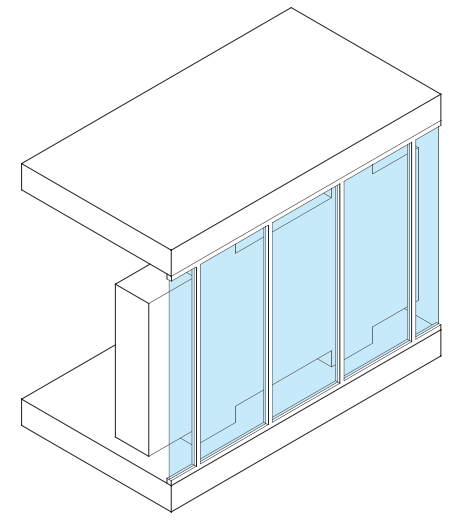

FIG. $21>$

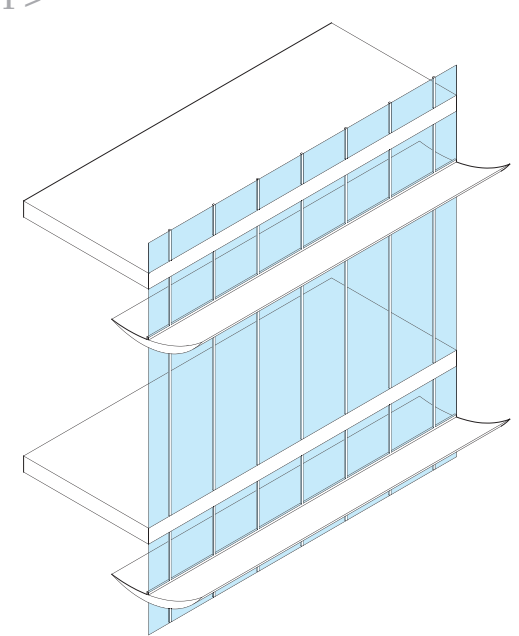

FIG.24 >

recibe la capa exterior. Esta estrategia amortigua el efecto del flujo solar (Qs) en elementos opacos y sirve como estrategia de control térmico en verano (FIG.22).

- Doble vidriado hermético (DVH): el uso de este tipo de componentes vidriados tiene el objetivo de controlar el flujo de transmisión (Qт) de calor a través de los elementos transparentes de la envolvente. Gracias a la cámara de aire, los DVH tienen un valor U muy inferior al de un vidrio simple y además es posible usar dobles o triples cámaras para llegar a valores más óptimos. Su combinación con vidrios reflectivos o absorbentes puede mejorar todavía más el desempeño de los componentes transparentes del sistema de cerramiento.

Control lumínico (Cl) / Tiene el objetivo objetivo de minimizar el uso de iluminación artificial, a través de una distribución del flujo luminoso que asegure que no se producirá deslumbramiento y que la iluminancia será suficiente para realizar las actividades propias del programa del edificio.

Entre las estrategias que permiten el control lumínico a través del sistema de cerramiento se encuentran: 

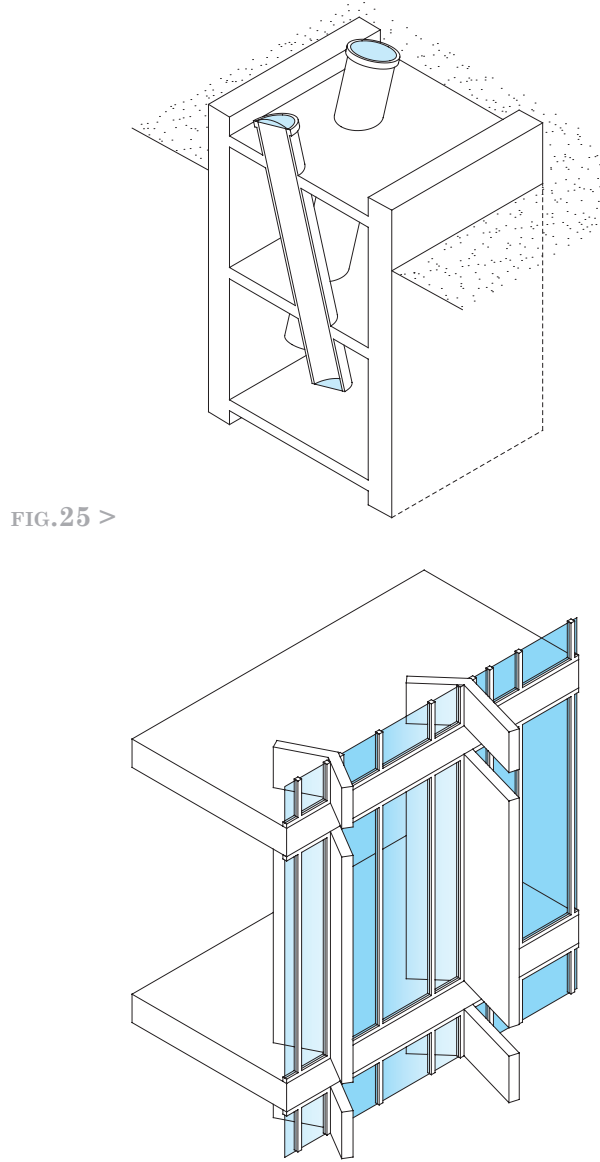

FIG. 13 Brise soleil.

Edificio CCU, +arquitectos, Santiago, 2003.

FIG.14 Aleros.

Edificio El Bosque 123, Murtinho \& Raby,

Santiago, 2009 .

FIG.15 Celosías.

Edificio Debis, Renzo Piano, Berlín, 1997.

FIG.16 Pantallas.

Hotel AC Córdoba, Isasi y Cáceres Arquitec-

tos, Córdoba, España, 2008

FIG.17 Doble piel.

Sede central de GSW, Sauerbuch \& Hutton, Berlín, 1999.

FIG.18 Doble piel corredor.

Stadtorr Düsseldorf, Petzinka \& Pink, Düsseldorf, 2002.

FIG.19 Doble piel cajón.

ARD-Hauptstadtstudio, Ortner \& Ortner

Baukunst, Berlín, 1999.

FIG.20 Doble piel shaft.

Photonics Center, Sauerbuch \& Hutton, Berlín, 1998.
FIC. 21 Muro trombe.

Sin datos de autor.

FIG.22 Fachada venilada.

Casa Pichara Saud, CVZARQ arquitectos, Santiago, 2005.

FIG. 23 Cortinas.

Edificio Mistral, A4 Arquitectos, Santiago, 2006 .

FIG.24 Bandejas de luz.

Edificio SOKA-BAU, Thomas Herzog, Wiesbaden, 2003

FIG.25 Conductos de sol.

Estación S-Bahn Postdamer Platz, Berlín, s/f

FIG.26 Planos reflectivos.

Dojo Toesca, CVZARQ arquitectos, Santiago, 2010 .

Fuente: elaboración propia.

- Cortinas: se pueden utilizar como cualquier sistema de control solar dispuesto en el interior, con el fin de manejar la componente lumínica de la radiación solar. Son útiles para evitar el deslumbramiento, sin embargo, estimulan el uso de iluminación artificial (FIG.23).

- Bandejas de luz: se utilizan para evitar el deslumbramiento por medio de la desviación de la fuente principal de iluminación hacia el cielo interior, lo que permite el uso de cortinas en la zona que ocupa el campo visual (FIG.24).

- Conductos de sol: sistema cuyo objetivo es llevar luz natural a lugares donde no llega o carece de suficiente intensidad. Consiste en una cabeza captadora que conduce la radiación a través de un tubo espejado, de máxima reflexión, hasta el lugar que requiere luz (FIG.25).

- Planos reflectivos: la manipulación de la forma de la envolvente permite el uso de planos destinados que utiliza luz natural para generar ambientes iluminados en los cuales se evita el deslumbramiento al sacar del campo visual la radiación solar directa (FIG.26).

CONCLUSIONES

El diseño de sistemas de cerramiento es complejo tanto por la cantidad de variables involucradas como por su carácter dinámico. Sin embargo no se trata de una complejidad asociada al cálculo, sino al dominio y la capacidad de percepción y predicción de conceptos como los aquí esbozados.

La aquí propuesta es una manera de enfrentar el diseño, atendiendo a las condiciones internas y externas, para evaluar estrategias de relación entre ellas a través del sistema de cerramiento. +

Bibliografía sugerida

D' ALENCON, Renato. Acondicionamientos: Arquitectura y Tecnica. Ediciones ARQ, Santiago, 2008, / DEPECKER, P.; Menezo, C.; virgone, J. y S. LEPERs. "Design of buildings shape and energetic consumption". Building and Environment, vol. 36, No 5. Elsevier, Maryland, junio de 2001, pp. 627-635. / REY MARTínez, Francisco. Eficiencia ernergética en edificios: certificación y auditorías energéticas. Thomson, Sidney, 2006. / SANTAMOURIS, M. "Heat-Island effect". asimakopoulos, D. Energy and Climate in the Urban Built Environment. James \& James, Londres, 2001. / TIBERIU, Catalina, VIRGONe Joseph y Eric BLANCO. "Development of a support tool for building design optimization and renewable energy integration", October 22nd to 24th 2008. Dublin. http:// architecture.ucd.ie/Paul/PLEA2008/content/papers/oral/PLEA_FinalPaper_ref_377.pdf. / YEANG, Ken. Proyectar Con La Naturaleza: Bases Ecológicas para el Proyecto Arquitectónico. Gustavo Gili, Barcelona, 1999. 